\title{
Inner Hair Cells Are Not Required for Survival of Spiral Ganglion Neurons in the Adult Cochlea
}

\author{
Yael Zilberstein, ${ }^{1,2}$ M. Charles Liberman, ${ }^{3,4}$ and Gabriel Corfas ${ }^{1,2,3}$ \\ ${ }^{1}$ F.M. Kirby Neurobiology Center, Children's Hospital Boston, Boston, Massachusetts 02115, Departments of ${ }^{2}$ Neurology and ${ }^{3}$ Otology and Laryngology, \\ Harvard Medical School, Boston, Massachusetts 02115, and ${ }^{4}$ Eaton-Peabody Laboratories, Massachusetts Eye \& Ear Infirmary, Boston, Massachusetts \\ 02114
}

Studies of sensorineural hearing loss have long suggested that survival of spiral ganglion neurons (SGNs) depends on trophic support provided by their peripheral targets, the inner hair cells (IHCs): following ototoxic drugs or acoustic overexposure, IHC death is rapid whereas SGN degeneration is always delayed. However, recent noise-trauma studies show that SGNs can die even when hair cells survive, and transgenic mouse models show that supporting cell dysfunction can cause SGN degeneration in the absence of IHC pathology. To reexamine this issue, we studied a model of IHC loss that does not involve noise or ototoxic drugs. Mice lacking the gene for the high-affinity thiamine transporter (Slc19a2) have normal cochlear structure and function when fed a regular (thiamine-rich) diet. However, dietary thiamine restriction causes widespread, rapid (within $10 \mathrm{~d}$ ) loss of IHCs. Using this model, we show that SGNs can survive for months after IHC loss, indicating that (1) IHCs are not necessary for neuronal survival, (2) neuronal loss in the other hearing loss models is likely due to effects of the trauma on the sensory neurons or other inner ear cells, and (3) that other cells, most likely supporting cells of the organ of Corti, are the main source of SGN survival factors. These results overturn a long-standing dogma in the study of sensorineural hearing loss and highlight the importance of cochlear supporting cells in neuronal survival in the adult inner ear.

\section{Introduction}

Degeneration of spiral ganglion neurons (SGNs), the primary sensory fibers of the auditory pathway, is an important component of sensorineural hearing loss. While the mechanisms that contribute to the long-term survival of SGNs in the normal and the injured ear remain poorly understood, the prevailing view is that SGN survival depends on ongoing trophic support provided by inner hair cells (IHCs). This idea is primarily based on three observations from animal models of acquired sensorineural hearing loss, such as acoustic trauma or ototoxic drugs: (1) loss of IHCs occurs rapidly (1-2 d) after exposure, whereas loss of SGNs (or their peripheral axons) occurs begins 3-15 d later (Dupont et al., 1993; McFadden et al., 2004; Gillespie and Shepherd, 2005), (2) the locations of SGN loss along the cochlear spiral correlate with the locations of IHC loss (Liberman and Kiang, 1978; Bohne and Harding, 2000), and (3) cochlear perfusion of neurotrophic factors, including NT-3, BDNF and GDNF, can prolong SGN survival for weeks to months after IHC loss (Gillespie and Shepherd, 2005).

Received Sept. 7, 2011; revised Nov. 2, 2011; accepted Nov. 2, 2011.

Author contributions: M.C.L. and G.C. designed research; Y.Z. performed research; M.C.L. and G.C. analyzed data; M.C.L. and G.C. wrote the paper.

This work was supported by National Institutes of Health Grants R01 DC0188 (M.C.L.), R01 DC04820 (G.C.), and P30 DC5029 (M.C.L.); the Department of Otolaryngology and Communication Enhancement, Children's Hospital Boston (G.C); and Intellectual and Developmental Disabilities Research Center NIH Grant P30-HD 18655 (G.C.). The skillful assistance of Leslie Dodds, Constance Miller, and Bethany Taylor is gratefully acknowledged.

Correspondence should be addressed to either of the following: Dr. Gabriel Corfas, F.M. Kirby Neurobiology Center, Children's Hospital Boston, 3 Blackfan Circle, CLS Room 12256,Boston MA 02115, E-mail: gabriel.corfas@childrens.harvard. edu; or Dr. M. Charles Liberman, Eaton-Peabody Laboratory, Massachusetts Eye \& Ear Infirmary, 243 Charles Street, Boston, MA 02114, E-mail: Charles_Liberman@meei.harvard.edu.

DOl:10.1523/JNEUROSCl.4678-11.2012

Copyright $\odot 2012$ the authors $\quad 0270-6474 / 12 / 320405-06 \$ 15.00 / 0$
However, recent studies suggest that supporting cells of the organ of Corti also play a central role in promoting SGN survival in the adult. For example, loss of ErbB receptor signaling in cochlear supporting cells leads to SGN degeneration after the onset of hearing in the absence of hair cell and supporting cell loss (Stankovic et al., 2004), indicating that normal supporting cell function is necessary for SGN survival. There is also evidence that the correlation between IHC loss and SGN death arises because both acoustic trauma and ototoxic drugs typically destroy most supporting cells in the regions where IHCs degenerate (Sugawara et al., 2005), and/or because the insult has direct effects on the neurons (Spoendlin, 1971; Liberman and Mulroy, 1982; Robertson, 1983). These studies, together with the observation that trophic factors known to promote SGN survival, such as NT3, are expressed by supporting cells (Sugawara et al., 2007), suggest that SGN survival in the adult cochlea may be mediated by supporting cells rather than hair cells. However, direct demonstration that SGN can survive for extended periods after IHC loss is lacking.

To directly test the role of IHCs in long-term SGN survival, we used a mouse model of IHC loss that does not involve noise or ototoxic drugs. Mice lacking the gene for the high-affinity thiamine (vitamin B1) transporter (Slc19a2) have normal cochlear structure and function when fed a regular (thiamine-rich) diet. However, dietary thiamine restriction causes widespread, rapid (within $10 \mathrm{~d}$ ) loss of IHCs, and the associated disappearance of cochlear neural responses in the adult (Liberman et al., 2006). Outer hair cells are spared in most cochlear locations. Here, we used this animal model to test whether loss of IHCs in this arguably more targeted with potentially less collateral 
damage to supporting cells and nerve terminals themselves, compromises long-term SGN survival in the adult.

\section{Materials and Methods}

Animals and groups

The mutant mouse line with targeted deletion of the high-affinity thiamine transporter (SLC19a2) was initially derived (Fleming et al., 2003) as a hybrid between C57BL/6 and 129 strains, and then backcrossed in our laboratory to $\mathrm{FVB} / \mathrm{N}$, genotyping at each generation to exclude animals carrying the hypomorphic cadherin 23 allele $\left(c d h 23^{753 \mathrm{~A}}\right)$ from $\mathrm{C} 57 \mathrm{BL} / 6$, associated with the age-related hearing loss phenotype. Wild-type mice and littermates homozygous for the thiamine transporter-null allele $\left(\right.$ Slc19a2 $\left.{ }^{-1-}\right)$ were maintained on a normal diet $(22 \mathrm{mg} / \mathrm{kg}$ thiamine) until 6 weeks of age. Then, a subset of wild-type and homozygous mutants were put on a low-thiamine diet $(2 \mathrm{mg} / \mathrm{kg})$ for $26 \mathrm{~d}$. Three months after the end of the $26 \mathrm{~d}$ thiamine restriction, animals were killed and subjected to histological analysis.

\section{Histological preparation}

Plastic sections. Animals were perfused intracardially with $2.5 \%$ glutaraldehyde and $1.5 \%$ paraformaldehyde in phosphate buffer. Cochleas were osmicated $\left(1 \% \mathrm{OsO}_{4}\right.$ in $\left.\mathrm{dH}_{2} \mathrm{O}\right)$, decalcified $(0.1 \mathrm{~m}$ EDTA with $0.4 \%$ glutaraldehyde), dehydrated in ethanols and propylene oxide, embedded in Araldite resins, and serially sectioned at $40 \mu \mathrm{m}$ in a quasi-horizontal plane.

Immunostaining. Mice were intracardially perfused with $4 \%$ paraformaldehyde in $0.1 \mathrm{~m}$ PBS, $\mathrm{pH}$ 7.4. Cochleas were dissected, postfixed overnight at $4^{\circ} \mathrm{C}$, and decalcified in $0.12 \mathrm{M}$ EDTA for $3 \mathrm{~d}$ at $4^{\circ} \mathrm{C}$. Cochleas were then either (1) cryoprotected in $30 \%$ sucrose overnight at $4^{\circ} \mathrm{C}$, embedded in OCT, and sectioned at $12 \mu \mathrm{m}$ or (2) dissected into half turns for whole-mount immunostaining. Antibodies included those directed against (1) heavy-neurofilaments (chicken anti-NFH, Millipore Bioscience Research Reagents, 1:1000), which stains afferent and efferent axons in the sensory epithelia, (2) vesicular acetylcholine transport [rabbit anti-VAT (vesicular acetylcholine transporter), Sigma, 1:1000], which label the cholinergic efferent innervation, and/or (3) glutamate transporter, which stains supporting cells in the IHC area (guinea pig anti-GLAST, Millipore Bioscience Research Reagents, 1:4000).

\section{Histological analysis}

Cochlear reconstructions. Cochlear location was specified for all morphological analyses so that place-matched cochlear regions were always compared. For whole mounts, lengths of all dissected pieces were measured by computerized planimetry. For serial-section material, 3-D reconstructions of the cochlear spiral were made using computer-aided anatomy software (Neurolucida), after which the relative position of each section through the cochlear spiral was computed. Cochlear location was converted into cochlear frequency with the map derived by Müller et al. (2005), as modified by Taberner and Liberman (2005).

Hair cell counts. Hair cells were counted in serial sections spanning the upper basal turn, centered on the $22 \mathrm{kHz}$ region, i.e., the same half-turn of the cochlea in which afferent innervation density was measured (see next paragraph). We choose this region because it is cut tangentially, thus allowing cross-sectional views of the peripheral axons of the cochlear nerve running radially in the osseous spiral lamina (Fig. $1 A, D$ ). Because the cutting plane is tangential, this half-turn of the cochlea is spanned in only six, serial $40 \mu \mathrm{m}$ sections. In each case, all six sections were examined using an oil-immersion lens [100×; numerical aperture (NA) 1.30] with differential interference contrast (DIC) optics: by focusing throughout the entire section thickness, accurate determinations of fractional loss of each cell type were made. By also counting inner and outer pillar cells as well as Deiters cells, which appeared to be present in normal numbers, the "expected" vs observed numbers of hair cells could be determined with a high degree of accuracy, even in these tangential sections.

Afferent innervation density. Axonal counts were made in crosssections through the osseous spiral lamina near the habenula perforata in tangential sections through the upper basal turn, i.e., in the same halfturn over which hair cells were counted. In each selected section, several fascicles of the peripheral axons of auditory nerve fibers were cut in cross section. All neuronal profiles in all fascicles in each section were counted using an oil-immersion lens (100×; NA 1.30) with DIC optics. To compute a fiber density, the number of fibers per fascicle was divided by the fascicle area.

Efferent innervation density. A semiquantitative analysis of innervation density of VAT-positive terminals in the IHC area was performed by an observer blind to the experimental groups. A four-point scale was used: $3=$ profuse, $2=$ moderate, $1=$ sparse, and $0=$ none.

\section{Results}

A group of young adult (6 weeks old) wild-type and slc19a2knock-out mice was fed a low-thiamine diet $(2 \mathrm{mg} / \mathrm{kg}$ thiamine $)$ for $26 \mathrm{~d}$, while another group was fed normal chow $(22 \mathrm{mg} / \mathrm{kg}$ thiamine). At the end of this period, a subset in each group (wildtype/high-thiamine, wild-type/low-thiamine, knock-out/highthiamine, and knock-out/low-thiamine) was randomly selected for evaluation of cochlear thresholds (auditory brainstem responses and distortion product otoacoustic emissions) and cochlear histopathology. As reported previously (Liberman et al., 2006), mutant mice on low-thiamine had sensory neuropathy caused by loss of almost all IHCs throughout the basal half of the cochlea, while most outer hair cells (OHCs) remained intact. In contrast, wild types in both high- and low-thiamine diet as well as mutant mice fed a highthiamine diet had normal thresholds and cochlear structure (data not shown). Remaining mice from the four groups were fed normal chow for an additional 3 months, a time at which the cochleas were removed and evaluated histologically.

To evaluate survival of hair cells and SGNs, serial plastic sections through the cochlear duct were prepared from 5-7 ears from each of the four groups (Fig. $1 \mathrm{~A}$, see schematic). The histological findings 3 months after termination of the low-thiamine challenge were virtually identical to those seen immediately after. As shown in Figure $1 \mathrm{~B}, \mathrm{KO}$ mice exposed to the low-thiamine diet showed massive degeneration of IHCs, while most of the $\mathrm{OHCs}$ and all the supporting cells remained intact and appeared normal in the light microscope. All accessory structures of the cochlear duct, including stria vascularis, spiral ligament, spiral limbus, tectorial membrane also appeared normal in the light-microscopic level. As also shown in prior reports (Liberman et al., 2006), there was no detectable histopathology when wild types were subjected to low thiamine, or when knock-outs were maintained completely on a high-thiamine regimen. Hair cell counts (Fig. 1C) show that $>95 \%$ of the IHCs were destroyed in the knock-out low-thiamine group, while $>66 \%$ of the $\mathrm{OHCs}$ remained intact. IHC and OHC losses were minimal in the other three groups.

The plastic sections were also used to examine the SGNs, the great majority of which make synaptic contact only with IHCs. As schematized in Figure $1 A$, the peripheral terminals (unmyelinated) of these so-called "type I" neurons are in the neuropil under the IHC, the peripheral axons (myelinated) are in the osseous spiral lamina, and their bipolar cell bodies are in the spiral ganglion. The micrographs in Figure $1 D$ show no obvious loss of SGNs in the ganglion, despite the longstanding and complete loss of IHCs in the same cochlear region. Furthermore, examination of the spiral ganglion with high-power objectives showed no signs of neurodegeneration (i.e., nuclear condensation or demyelination) in the $\mathrm{KO} /$ low-thiamine groups. As the cell bodies are difficult to count in these relatively thick plastic sections, and because the peripheral axons of SGNs degenerate well before their cell bodies, we chose to quantify the neuronal loss by counting peripheral axons. As illustrated in Figure $1 F$, these myelinated axons can be clearly resolved and easily counted in osmiumstained sections oriented tangentially to the cochlear spiral, which cut the osseous spiral lamina in cross-section (see schema- 
A
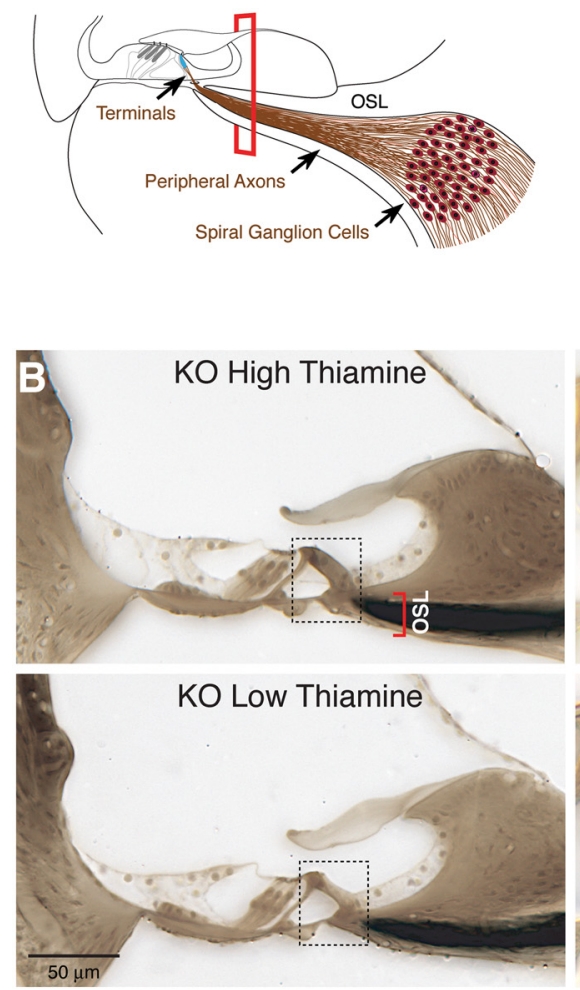

C

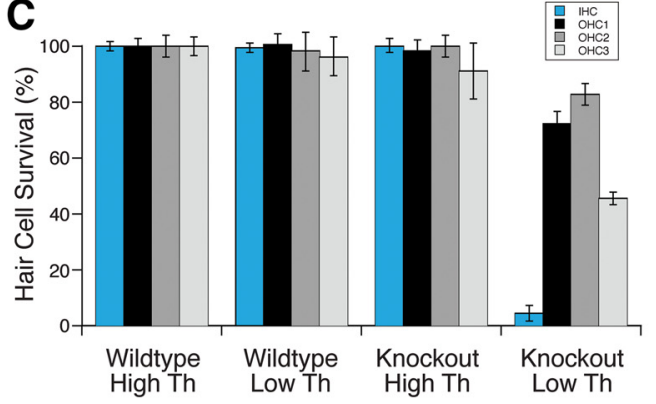

E

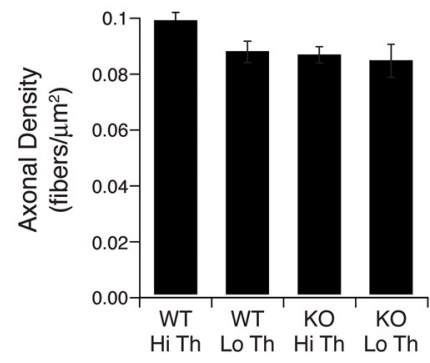

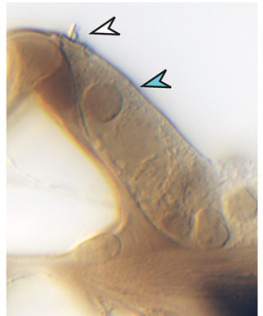
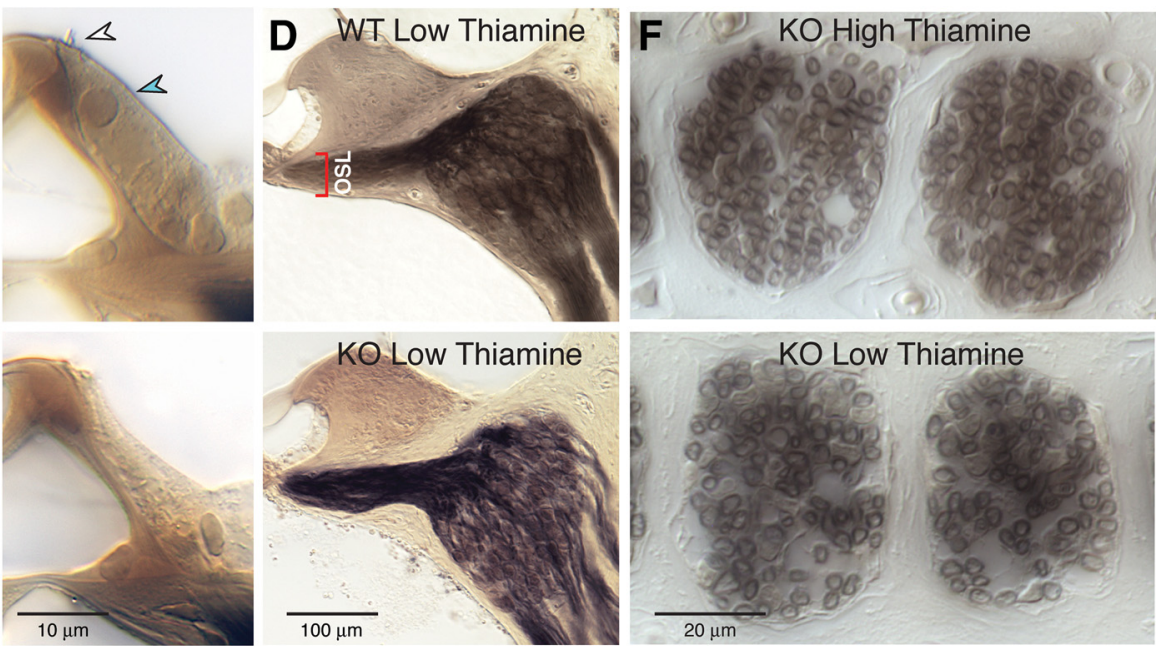

Figure 1. Three months after IHC loss, there is no significant loss of cochlear neurons in the SIC19a2-null mice on low thiamine. A, Schematic cross-section through the cochlear duct illustrating the cell bodies of SGNs, their peripheral axons and terminals. The red outline schematizes the section plane through the osseous spiral lamina (OSL) tangential to the cochlear spiral in which SGN peripheral axons are quantified $(\boldsymbol{E}, \boldsymbol{F}) . \boldsymbol{B}$, Photomicrographs of the midbasal turn of the cochlear duct ( $\sim 32 \mathrm{kHz}$ region) from a SIc19a2-null mouse on high thiamine (top row-IHCs present) and a knock-out mouse on low thiamine (bottom row - IHCs absent). In the high-power views at the right, the blue arrowhead points to the IHC nucleus; the white arrowhead points to the IHC hair bundle. Scale bars apply to both micrographs in each column. C, Inner and outer hair cells were counted throughout the upper basal turn in 5-7 ears from each of 4 experimental groups: wild types on high-thiamine, wild types on low thiamine, knock-outs on high thiamine and knock-outs on low thiamine. Means and SEs are shown. D, Photomicrographs of the midbasal turn ( $\sim 32 \mathrm{kHz}$ region) show that loss of IHCs in the knock-out mouse on low thiamine (bottom) does not obviously affect SGN density, when compared with the wild-type (top), in which the IHCs are present. Scale bar applies to both panels. $\boldsymbol{E}$, Peripheral axons were counted in the upper basal turn in 5-7 ears from each of the 4 experimental groups. Means and SEs are shown. $\boldsymbol{F}$, Photomicrographs of the upper basal turn ( $\sim 22 \mathrm{kHz}$ region) show SGN peripheral axons as they appear in cross-sections through the osseous spiral lamina from knock-out mice on high- vs low-thiamine diets.

tized section plane in Fig. $1 A$ ). As shown in Figure $1 E$, the neuronal counts were not significantly different across the four experimental groups, despite the essentially complete loss of IHCs in this region in the $\mathrm{KO} /$ low-thiamine group.

To further assess the supporting cells and the survival of the unmyelinated terminals of afferent and efferent fibers in the IHC area, 6-9 ears from a second set of knock-outs and wild types on low-vs high-thiamine were processed for immunostaining, three months after termination of the low-thiamine challenge. Our prior work suggested that the inner border and inner phalangeal cells (see schematic in Fig. 2A), which surround the IHC and the unmyelinated terminals of SGNs, are particularly important for the survival of cochlear neurons (Stankovic et al., 2004; Sugawara et al., 2005). We therefore used immunostaining for GLAST, a glutamate transporter strongly expressed by both inner border and inner phalangeal cells, which clears the glutamate released from the IHC/type-I synapses (Hakuba et al., 2000; Glowatzki et al., 2006). Comparison of the immunostained sections (Fig. $2 B$ ) and whole mounts (Fig. 2E) shows that in all cases, despite the longstanding loss of IHCs, the remaining supporting cells continue to show robust GLAST expression, suggesting that protein synthesis is not severely compromised.

To assess the unmyelinated nerve terminals in the IHC neuropil, we immunostained for neurofilament proteins. Remarkably, stain- ing of frozen sections showed that, three months after complete IHC loss, peripheral terminals of cochlear neurons remain in the IHC area, still surrounded by GLAST-positive supporting cells (Fig. 2B). The afferent nature of these neurofilament-positive fibers is confirmed by their radial rather than spiral orientation. Finally, to examine the survival of the efferent innervation in the IHC area, we immunostained cochlear whole mounts for a cholinergic marker (VAT), which labels the great majority of olivocochlear (OC) efferent fibers projecting to either IHC or OHC areas (Darrow et al., 2006). The subset of OC fibers projecting to the IHC area, i.e., the lateral OC (LOC) efferents, normally spiral in the inner spiral bundles, just underneath (and around the bases of) the IHCs (Fig. 2A), making synapses on numerous type-I dendrites via en passant contacts (Liberman, 1980; Brown, 1987). These spiral bundles are well seen in immunostained whole mounts of the organ of Corti (Fig. $2 D$ ). Blinded semiquantitative analysis of the samples suggested that LOC innervation density was unchanged among all four experimental groups (Fig. 2C).

\section{Discussion}

Numerous animal studies of acquired sensorineural hearing loss have shown that noise or ototoxic drugs can cause rapid hair cell loss followed by SGN degeneration (Liberman and Kiang, 1978; Lawner et al., 1997; Bohne and Harding, 2000; Gillespie and 

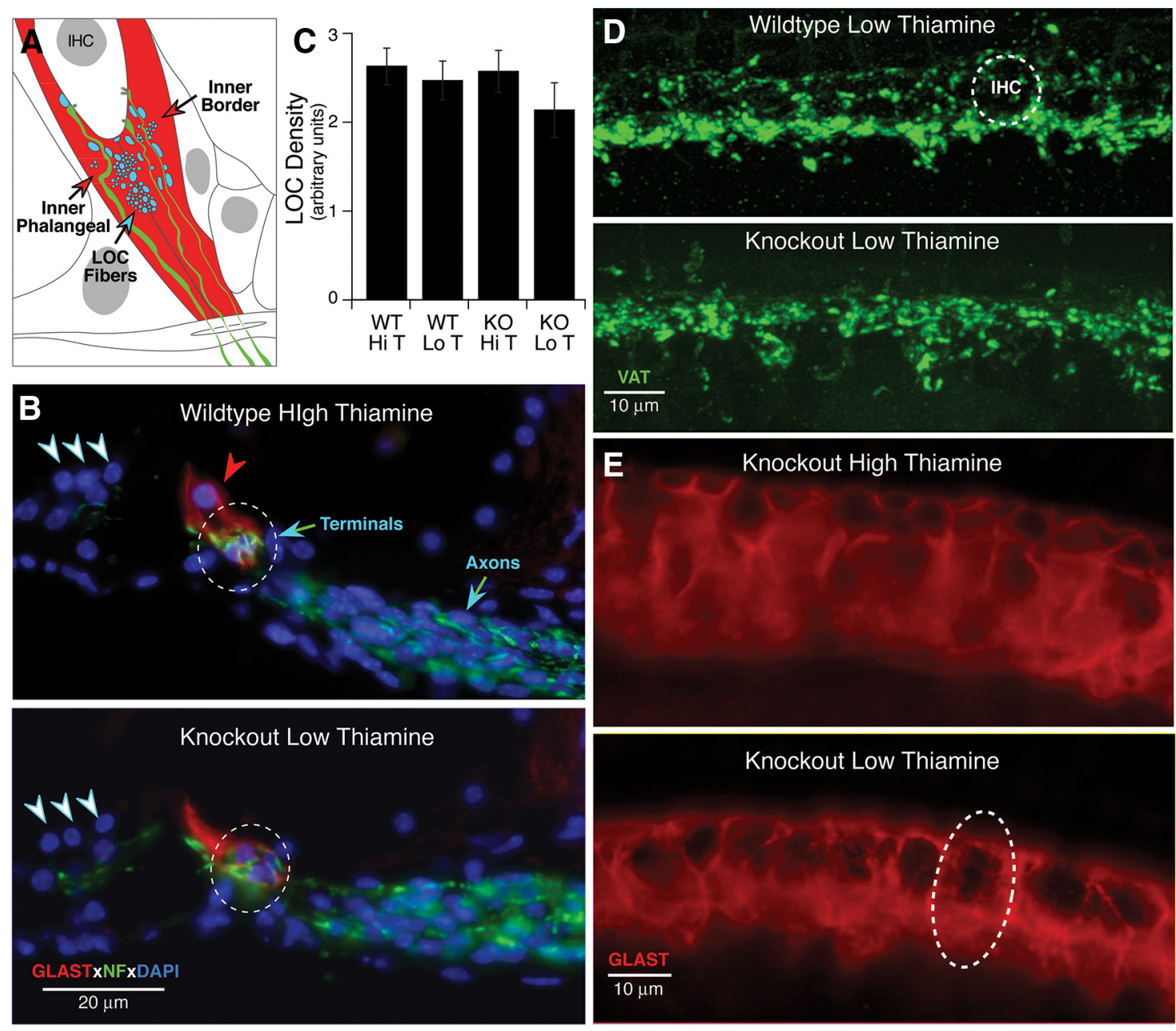

Figure 2. Three months after IHC loss, supporting cells, as well as afferent and efferent nerve terminals in the IHC area remain intact. $A$, Schematic of the IHC area illustrating SGN peripheral terminals, LOC efferent terminals and the two cells strongly expressing GLAST protein (inner border and inner phalangeal cells). $\boldsymbol{B}$, Confocal micrographs of immunostained sections through the organ of Corti and osseous spiral lamina from the midbasal turn ( $\sim 32 \mathrm{kHz}$ region). Three rows of $\mathrm{OHCs}$ are indicated by the white arrowheads in each panel. The DAPI-stained IHC nucleus (top only) is indicated by the red arrowhead. Dashed circles outline the neuropil in the IHC region where the unmyelinated SGN terminals are visible in both panels. The staining key and scale bar apply to both panels. C, Semiquantitative evaluation of LOC innervation density in confocal images such as those in $\boldsymbol{D}$, acquired from ears in the four experimental groups. Means and SEs are shown. $\boldsymbol{D}$, Maximum projections from confocal $z$-stacks of the VAT-stained LOC terminals in the IHC area. The approximate size of an IHC is indicated by the dashed circle in top panel. Scale applies to both panels. $\boldsymbol{E}$, Maximum projections from confocal $z$-stacks through whole-mounts of the organ of Corti in the IHC area immunostained with an anti-GLAST antibody. The spaces where the IHCs used to be are visible in the knock-out ear maintained on low thiamine (dashed circle indicates one space). Key and scale bar applies to both panels.

Shepherd, 2005). The similarity in spatial patterning of hair cell and neuronal death, combined with the delay in loss of SGNs, has led to the belief that cochlear nerve degeneration is a consequence of hair cell death and is due to the loss of some hair-cell generated trophic factor. Our results show that, in contrast to neural degeneration patterns after ototoxic drugs or acoustic trauma, SGNs survive, and even maintain their peripheral axon projections into the organ of Corti, long after IHCs loss, when the IHC loss is caused by another mechanism, i.e., thiamine transport deficiency. These findings suggest that the neuronal loss caused by ototoxic drugs or acoustic trauma arises from dysfunction of other cells within the inner ear that are involved in promoting the long-term survival of SGNs and/or that these insults directly damage the neurons themselves.

With respect to acoustic overstimulation, the idea of direct effects on the nerve terminals is well established: terminals swell and rupture immediately after exposure, even in cases that will not cause hair cell death (Liberman and Mulroy, 1982; Robertson, 1983), and a recent study has shown that acoustic overexposure can kill SGNs even if the IHCs survive (Kujawa and
Liberman, 2009). In the case of the ototoxic drug carboplatin, an ultrastructural study has shown that degenerative changes in the cochlear nerve terminals are visible within hours after treatment, even before ultrastructural changes are detectable in the IHCs (Wang et al., 2003). Similarly, an ultrastructural study of aminocoglycoside treatment has shown that nerve terminals swell within 15-18 h post-treatment, at about the same time that hair cells begin to be extruded from the epithelium (Hirose et al., 2004). We propose that IHC loss in the present study does not lead to SGN death because, here, IHCs die in a controlled fashion, presumably via apoptosis, without causing excess glutamate release and associated postsynaptic glutamate excitotoxicity, that may occur during overexposure to either intense sound or ototoxic drugs (Wang et al., 2003). It is not clear why IHCs are particularly vulnerable to thiamine deficiency, but expression of a second transporter by other cell types of the organ of Corti is a possible scenario.

The neuronal loss from ototoxic drugs or acoustic trauma might also result from associated loss of the IHC supporting cells. There is accumulating evidence that these supporting cells, i.e., 
the inner border and inner phalangeal cells, are key to neuronal survival in the adult ear. These supporting cells are in intimate contact with the unmyelinated terminals of cochlear nerve fibers in the neuropil underneath the IHCs. In the adult cochlea, these supporting cells express NT-3 (Sugawara et al., 2007), a neurotrophic factor necessary for SGN survival during development (Fariñas et al., 1994) and capable of enhancing SGN survival after IHC loss when perfused into the cochlea (Miller et al., 1997). Alterations in ErbB receptor signaling in supporting cells results in reduced NT3 expression accompanied by dramatic SGN degeneration and hearing loss without alterations in the number or appearance of either supporting cells or hair cells (Stankovic et al., 2004). Last, histological studies show a strong correlation between supporting cells loss and SGN death, both in animal models (Sugawara et al., 2005) and in human temporal bone studies (Suzuka and Schuknecht, 1988). Together, these results suggest that SGN survival in the adult cochlea may be mediated by supporting cells rather than hair cells.

In the IHC loss model described here, the sensitivity of IHCs to high-affinity thiamine transporter dysfunction appears to avoid the combined effects of IHC death, supporting cell damage and/or direct damage to the neurons seen in other models of cochlear pathology. In other hair-cell loss models, disappearance of SGN peripheral axons is first visible 1-2 weeks after cochlear insult, and SGN death (and associated loss of the central axons) progresses dramatically over 1-3 months. These patterns have been reported after acoustic trauma (Spoendlin, 1975; Liberman and Kiang, 1978; Wang et al., 2002), where SGN loss is virtually total 3 months after exposure (Spoendlin, 1975). After aminoglycoside antibiotics, SGN loss approaches $95 \%$ by 2 months posttreatment (Gillespie and Shepherd, 2005). After carboplatin treatment, SGNs are reduced by $50 \%$ at 12 weeks post-treatment (Takeno et al., 1998). In a well studied form of congenital hearing loss, the Bronx Waltzer mouse, IHCs degenerate in the neonatal period and $50 \%$ of the SGN peripheral axons are gone by 4 weeks of age (Schrott et al., 1989). The normal neuronal counts in the present study at 4 months after the onset of hair cell loss stand in stark contrast to the degenerative time course seen in all other studies and provide strong evidence that IHCs are not required for SGN survival.

Defining the mechanisms that promote survival of SGNs is relevant to human health and the treatment of hearing loss due to IHC degeneration. While some histopathological studies in temporal bones of humans with hearing loss reported that SGN loss is seen if and only if there is significant IHC loss in the same cochlear region (Johnsson, 1974; Felix et al., 2002), others showed that SGN death is more pronounced if supporting cells are also destroyed areas of IHC loss (Suzuka and Schuknecht, 1988), an observation similar to the one we made in animal models (Sugawara et al., 2005). The widespread success of cochlear implants, which bypass the cochlear sensory cells to directly stimulate cochlear neurons in profoundly deaf ears (Rubinstein, 2004), suggests that, in many types of acquired or congenital sensorineural hearing loss, SGNs often survive despite widespread loss of cochlear sensory cells. In this way, results from this novel mouse model of hearing loss fit well with clinical findings, suggesting that supporting cells of the human ear might play key roles in the long-term maintenance of SGNs. This mouse model could also be useful in testing new approaches to hair cell replacement, since all other structures of the organ of Corti remain intact, including neuronal terminals at a site where they could reinnervate newly generated sensory cells.

\section{References}

Bohne BA, Harding GW (2000) Degeneration in the cochlea after noise damage: primary versus secondary events. Am J Otol 21:505-509.

Brown MC (1987) Morphology of labeled efferent fibers in the guinea pig cochlea. J Comp Neurol 260:605-618.

Darrow KN, Simons EJ, Dodds L, Liberman MC (2006) Dopaminergic innervation of the mouse inner ear: evidence for a separate cytochemical group of cochlear efferent fibers. J Comp Neurol 498:403-414.

Dupont J, Guilhaume A, Aran JM (1993) Neuronal degeneration of primary cochlear and vestibular innervations after local injection of sisomicin in the guinea pig. Hear Res 68:217-228.

Fariñas I, Jones KR, Backus C, Wang XY, Reichardt LF (1994) Severe sensory and sympathetic deficits in mice lacking neurotrophin-3. Nature 369:658-661.

Felix H, Pollak A, Gleeson M, Johnsson LG (2002) Degeneration pattern of human first-order cochlear neurons. Adv Otorhinolaryngol 59:116-123.

Fleming JC, Tartaglini E, Kawatsuji R, Yao D, Fujiwara Y, Bednarski JJ, Fleming MD, Neufeld EJ (2003) Male infertility and thiamine-dependent erythroid hypoplasia in mice lacking thiamine transporter Slc19a2. Mol Genet Metab 80:234-241.

Gillespie LN, Shepherd RK (2005) Clinical application of neurotrophic factors: the potential for primary auditory neuron protection. Eur J Neurosci 22:2123-2133.

Glowatzki E, Cheng N, Hiel H, Yi E, Tanaka K, Ellis-Davies GC, Rothstein JD, Bergles DE (2006) The glutamate-aspartate transporter GLAST mediates glutamate uptake at inner hair cell afferent synapses in the mammalian cochlea. J Neurosci 26:7659-7664.

Hakuba N, Koga K, Gyo K, Usami SI, Tanaka K (2000) Exacerbation of noise induced hearing loss in mice lacking the glutamate transporter GLAST. J Neurosci 20:8750-8753.

Hirose K, Westrum LE, Cunningham DE, Rubel EW (2004) Electron microscopy of degenerative changes in the chick basilar papilla after gentamicin exposure. J Comp Neurol 470:164-180.

Johnsson LG (1974) Sequence of degeneration of Corti's organ and its firstorder neurons. Ann Otol Rhinol Laryngol 83:294-303.

Kujawa SG, Liberman MC (2009) Adding insult to injury: cochlear nerve degeneration after "temporary" noise-induced hearing loss. J Neurosci 29:14077-14085.

Lawner BE, Harding GW, Bohne BA (1997) Time course of nerve-fiber regeneration in the noise-damaged mammalian cochlea. Int J Dev Neurosci 15:601-617.

Liberman MC (1980) Efferent synapses in the inner hair cell area of the cat cochlea: an electron microscopic study of serial sections. Hear Res 3:189-204.

Liberman MC, Kiang NY (1978) Acoustic trauma in cats. Cochlear pathology and auditory-nerve activity. Acta Otolaryngol Suppl 358:1-63.

Liberman MC, Mulroy MJ (1982) Acute and chronic effects of acoustic trauma: Cochlear pathology and auditory nerve pathophysiology. In: New perspectives on noise-induced hearing loss (Hamernik RP, Henderson D, Salvi R, eds), pp 105-136. New York: Raven.

Liberman MC, Tartaglini E, Fleming JC, Neufeld EJ (2006) Deletion of SLC19A2, the high affinity thiamine transporter, causes selective inner hair cell loss and an auditory neuropathy phenotype. J Assoc Res Otolaryngol 7:211-217.

McFadden SL, Ding D, Jiang H, Salvi RJ (2004) Time course of efferent fiber and spiral ganglion cell degeneration following complete hair cell loss in the chinchilla. Brain Res 997:40-51.

Miller JM, Chi DH, O'Keeffe LJ, Kruszka P, Raphael Y, Altschuler RA (1997) Neurotrophins can enhance spiral ganglion cell survival after inner hair cell loss. Int J Dev Neurosci 15:631-643.

Müller M, von Hünerbein K, Hoidis S, Smolders JW (2005) A physiological place-frequency map of the cochlea in the CBA/J mouse. Hear Res 202:63-73.

Robertson D (1983) Functional significance of dendritic swelling after loud sounds in the guinea pig cochlea. Hearing Res 9:263-278.

Rubinstein JT (2004) How cochlear implants encode speech. Curr Opin Otolaryngol Head Neck Surg 12:444-448.

Schrott A, Stephan K, Spoendlin H (1989) Hearing with selective inner hair cell loss. Hear Res 40:213-219. 
Spoendlin H (1971) Primary structural changes in the organ of Corti after acoustic overstimulation. Acta Otolaryngol 71:166-176.

Spoendlin H (1975) Retrograde degeneration of the cochlear nerve. Acta Otolaryngol 79:266-275.

Stankovic K, Rio C, Xia A, Sugawara M, Adams JC, Liberman MC, Corfas G (2004) Survival of adult spiral ganglion neurons requires erbB receptor signaling in the inner ear. J Neurosci 24:8651-8661.

Sugawara M, Corfas G, Liberman MC (2005) Influence of supporting cells on neuronal degeneration after hair cell loss. J Assoc Res Otolaryngol 6:136-147.

Sugawara M, Murtie JC, Stankovic KM, Liberman MC, Corfas G (2007) Dynamic patterns of neurotrophin 3 expression in the postnatal mouse inner ear. J Comp Neurol 501:30-37.
Suzuka Y, Schuknecht HF (1988) Retrograde cochlear neuronal degeneration in human subjects. Acta Otolaryngol Suppl 450:1-20.

Taberner AM, Liberman MC (2005) Response properties of single auditory nerve fibers in the mouse. J Neurophysiol 93:557-569.

Takeno S, Wake M, Mount RJ, Harrison RV (1998) Degeneration of spiral ganglion cells in the chinchilla after inner hair cell loss induced by carboplatin. Audiol Neurootol 3:281-290.

Wang J, Ding D, Salvi RJ (2003) Carboplatin-induced early cochlear lesion in chinchillas. Hear Res 181:65-72.

Wang Y, Hirose K, Liberman MC (2002) Dynamics of noise-induced cellular injury and repair in the mouse cochlea. J Assoc Res Otolaryngol 3:248-268. 